\title{
Hybrid Spinel Iron Oxide Nanoarchitecture Combining Crystalline and Amorphous Parent Material
}

\author{
Sawssen Slimani ${ }^{1,3,6}$, Giorgio Concas², Francesco Congiu², Gianni Barucca ${ }^{4}$, \\ Nader Yaacoub ${ }^{5}$, Alessandro Talone ${ }^{3,7}$, Mourad Smari ${ }^{6}$, Essebti Dhahri ${ }^{6}$ Davide \\ Peddis $^{1,3}$, Giuseppe Muscas ${ }^{2 *}$ \\ ${ }^{1}$ Dipartimento di Chimica e Chimica Industriale, Università degli Studi di Genova, Via \\ Dodecaneso 31, 1-16146 Genova, Italy. \\ ${ }^{2}$ Department of Physics, University of Cagliari, Cittadella Universitaria di Monserrato, S.P. $8 \mathrm{Km}$ \\ 0.700, I-09042 Monserrato (CA), Italy. \\ ${ }^{3}$ Istituto di Struttura della Materia-CNR, 00015 Monterotondo Scalo (RM), Italy. \\ ${ }^{4}$ Dipartimento di Scienze e Ingegneria della Materia, dell'Ambiente ed Urbanistica, Università \\ Politecnica delle Marche, Via Brecce Bianche 12, 60131 Ancona, Italy. \\ 5IMMM, Université du Main, CNRS UMR-6283, Avenue Olivier Messiaen, Le Mans 72085, France. \\ ${ }^{6}$ Laboratoire de Physique Appliquée, Faculté des Sciences, Université de Sfax, B.P. 1171, 3000 \\ Sfax, Tunisia. \\ ${ }^{7}$ Dipartimento di Scienze, Università degli Studi 'Roma Tre', 00154 Roma, Italy
}

\section{Supporting Information}

\footnotetext{
* Corresponding author e-mail: giuseppe.muscas@dsf.unica.it
} 


\section{X-Ray Diffraction analysis}

Figure S1 reports the XRD patterns of two samples, the nanocomposite ( $\mathrm{CoFe}_{2} \mathrm{O}_{4}+$ amorphous) and the $\mathrm{Co}_{0.25} \mathrm{Fe}_{2.75} \mathrm{O}_{4}$ sample, prepared at a $\mathrm{pH}=9.5$ and constant temperature of $60^{\circ} \mathrm{C}$. The intensity of the peaks increases and shows better crystallinity for the $\mathrm{Co}_{0.25} \mathrm{Fe}_{2.75} \mathrm{O}_{4}$ sample. Indeed, its average crystalline particle size, estimated using the Scherrer formula, exhibits a value of $\approx 7 \mathrm{~nm}$, clearly larger than $\approx 3 \mathrm{~nm}$ obtained for the nanocomposite ( $\mathrm{CoFe}_{2} \mathrm{O}_{4}+$ amorphous). During the synthesis process, the solution exhibited a reddish-brown color, indicating the presence of some cobalt as $\mathrm{Co}\left(\mathrm{H}_{2} \mathrm{O}\right)_{4}{ }^{2+}$ forming a non-stoichiometric hydroxide precipitate. At a $\mathrm{pH}$ of 9.5, the hydrolysis constants of iron species are much larger than those of cobalt species, which lead to a reduction in the amorphous phase and larger crystalline domains of the final product as the amount of $\mathrm{Co}^{2+}$ decreases ${ }^{1}$. Therefore, the obtained results suggest that the precipitation of cobalt species in an aqueous solution needs a $\mathrm{pH}$ value higher than that of the iron species ${ }^{2}$.

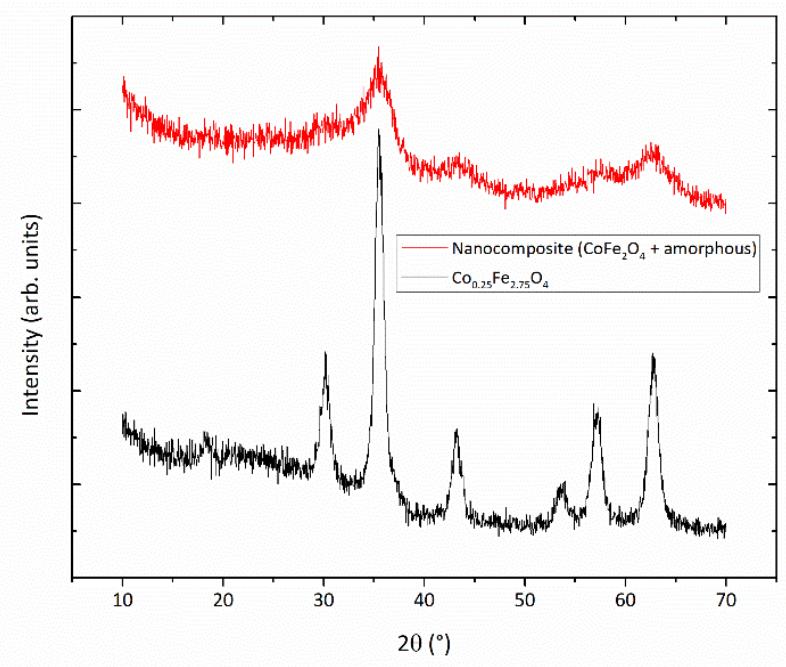

Figure S1. XRD patterns of the nanocomposite ( $\mathrm{CoFe}_{2} \mathrm{O}_{4}+$ amorphous) and the $\mathrm{Co}_{0.25} \mathrm{Fe}_{2.75} \mathrm{O}_{4}$ sample, prepared at $\mathrm{pH}=9.5$ and at a constant temperature of $60^{\circ} \mathrm{C}$. 
Transmission electron microscopy analysis

a

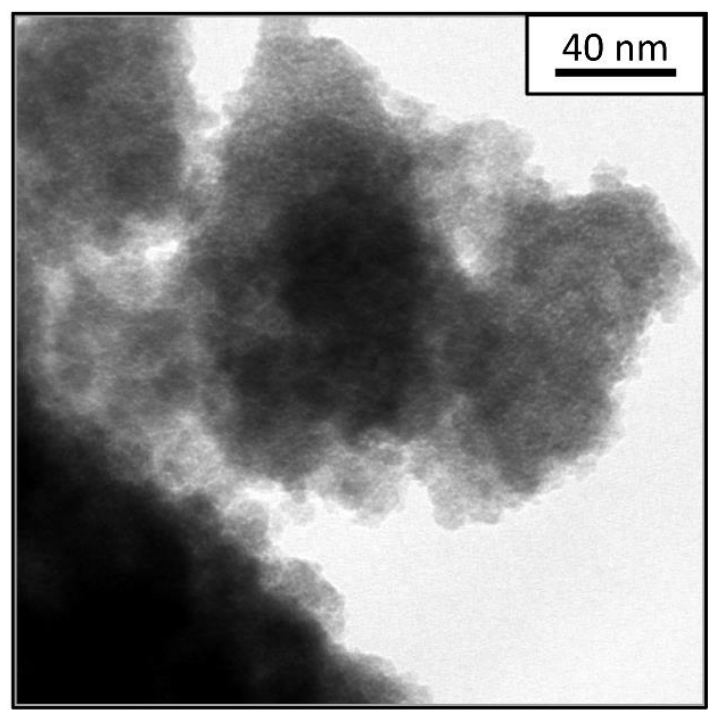

b

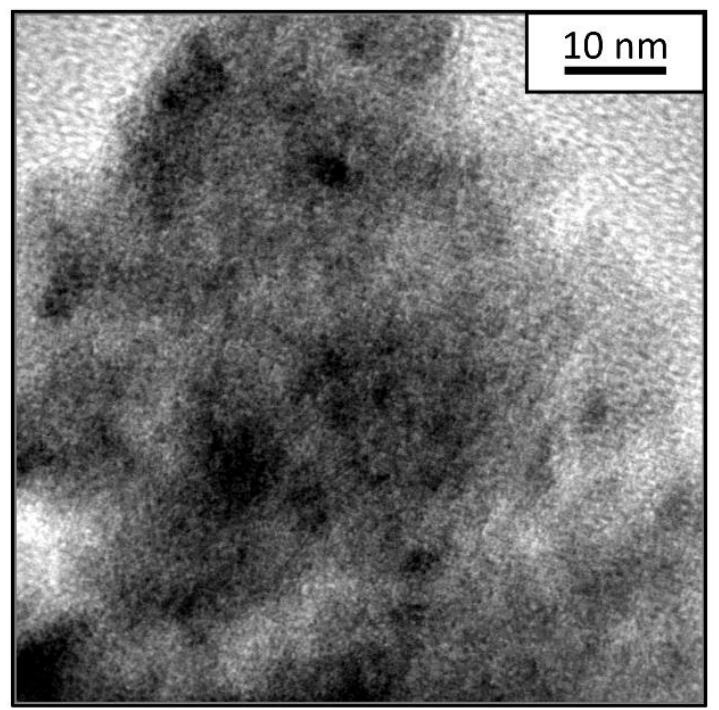

Figure S2: Bright-field TEM images of the nanocomposite (CoFe $\mathrm{O}_{4}+$ amorphous) taken at different magnifications, (a) and (b), showing the large aggregates with a granular structure that compose the sample. 


\section{High field Mössbauer spectroscopy}

${ }^{57} \mathrm{Fe}$ Mössbauer spectra have been recorded at $10 \mathrm{~K}$ under a magnetic field of $8 \mathrm{~T}\left(\mathrm{~B}_{\text {ext }}\right)$ applied parallel to the $\gamma$ -beam. When the second and fifth lines have a non-zero intensity, they evidence a non-collinear structure for iron magnetic moments with respect to the applied field ${ }^{3,4}$. In the case of a non-collinear spin structure, the measured effective nucleus field $\left(B_{\text {eff }}\right)$ differs from the external field $\left(B_{\text {ext }}\right)$ due to the average canting angle $\theta$ as graphically illustrated in Figure S3.

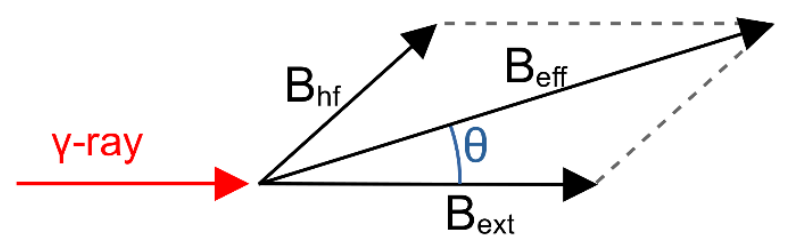

Figure S3. A sketch of the geometrical arrangement of the setup for ${ }^{57} \mathrm{Fe}$ Mössbauer spectroscopy and the relation between the hyperfine field $\left(B_{h f}\right)$, the measured effective nucleus field $\left(B_{\text {eff }}\right)$, and the external field $\left(B_{\text {ext }}\right)$, forming the average canting angle $\vartheta$.

For a thin sample, where thickness effects are negligible and where the direction of the hyperfine field is at random with respect to the $\gamma$-ray direction, the relative area of the Zeeman sextet are in the ratio:

$$
3: 2 p: 1: 1: 2 p: 3
$$

where $p$ is dependent on the canting angle $\theta$ :

$$
p=\frac{2 \sin ^{2} \theta}{1+\cos ^{2} \theta}
$$

By normalizing the total area to 1 , the area of lines $2-5\left(A_{2,5}\right)$ is equal to:

$$
A_{2,5}=\frac{1}{2} \sin ^{2} \theta
$$

In the absence of canting effects, lines 2-5 disappear. On the other hand, from their residual intensity, the corresponding average canting angle $\theta$ over the entire particle is determined ${ }^{3}$ :

$$
\theta=\arcsin \sqrt{2 A_{2,5}}
$$




\section{Analysis of $T_{B}(H)$ : random anisotropy model (RAM)}

For interacting magnetic nanoparticles, the effective anisotropy results from the average contribution of the individual interacting elements in a so-called random anisotropy model (RAM). The model has been largely demonstrated for exchange-coupled grains ${ }^{5}$. Recently, it has been successfully proposed to investigate the interplay between the individual particles anisotropy energy and the interparticle interactions for purely dipolar coupled particles ${ }^{6}$.

For an ensemble of non-interacting particles, the dependence of $T_{B}$ on the applied field $H$ is described by the law 7,8 :

$$
T_{B}=\frac{K V}{k_{B} \ln \left(\frac{\tau_{m}}{\tau_{0}}\right)}\left[1-\frac{\mu_{0} H}{\mu_{0} H_{K}}\right]^{1.5}
$$

where $\mathrm{K}$ is the intrinsic anisotropy constant of the material, $\mathrm{V}$ the nanoparticle volume, $\tau_{\mathrm{m}} \sim 60 \mathrm{~s}$ (i.e., the typical experimental time in SQUID dc magnetization measurements), $\tau_{0}=10^{-11} \mathrm{~s}$ (commonly used for ferromagnetic particles), $\mathrm{H}_{\mathrm{K}}$ the anisotropy field, $\mu_{0}=4 \pi 10^{-7} \mathrm{H} / \mathrm{m}$ the vacuum permeability, and $\mathrm{k}_{\mathrm{B}}=1.3806510^{-23} \mathrm{~J} / \mathrm{K}$ is the Boltzmann constant. For ensembles of interacting nanoparticles, their interactions extend over a magnetic correlation length $\left(\mathrm{L}_{\text {corr }}\right)^{9}$ that can be expressed as a function of the applied field ${ }^{8,10}$ :

$$
L_{c o r r}=D+\left[\frac{2 A_{e f f}}{M_{S} \mu_{0} H+C}\right]^{1 / 2}
$$

where $A_{\text {eff }}$ represents an effective interaction intensity ${ }^{5}, D$ is the average particle diameter, and $M_{s}$ the saturation magnetization. The parameter $\mathrm{C}$ is needed to solve the divergence at $\mu_{0} \mathrm{H}=0 \mathrm{~T}$. It considers the influence of particles concentration on interactions, assuming a value close to zero for clustered particles and the form $C \approx$ $2 A_{\text {eff }}-M_{S} \mu_{0} H$ for non-interacting ones ${ }^{7,8}$. $L_{\text {corr }}$ defines the physical extension of the interactions. One can determine the number of correlated particles considering the volume fraction $x$ effectively occupied by the particles in the ensemble:

$$
N=1+x \frac{\left(L_{\text {corr }}^{3}-D^{3}\right)}{D^{3}}
$$

Therefore, those $\mathrm{N}$ particles form an effective volume $\mathrm{V}_{\mathrm{N}}$ of magnetic material interacting within the correlation length $\mathrm{L}_{\text {corr: }}$ :

$$
V_{N}=\frac{\pi}{6}\left[D^{3}+x\left(L_{\text {corr }}^{3}-D^{3}\right)\right]
$$

Within this correlation volume the effective anisotropy constant $K_{\text {eff }}$ results from the random walk effect of the anisotropy of the $\mathrm{N}$ correlated particles ${ }^{5}$ :

$$
K_{e f f}=\frac{K_{a}}{\sqrt{N}}
$$

As the interparticle interactions increase, the correlation length expands and the anisotropy averages out over a larger volume, thus reducing its effective magnitude. On the other hand, an external magnetic field reduces the correlation length ${ }^{10}$. For an ensemble of interacting particles, one should consider the effective anisotropy field $\mu_{0} \mathrm{H}_{\mathrm{K}}{ }^{\mathrm{N}}$ of the cluster of $\mathrm{N}$ correlated particles, which is linked to its effective average anisotropy $\mathrm{K}_{\text {eff }}{ }^{7,8}$ : 


$$
\mu_{0} H_{K}^{N}=2 \frac{K_{e f f}}{M_{S}}
$$

Finally, equation (5) can be re-written by substituting $\mathrm{K}, \mathrm{V}$, and $\mathrm{H}_{\mathrm{K}}$ with the effective values for the cluster $\mathrm{K}_{\text {eff, }}$ $V_{N}$, and $H_{K}{ }^{N}$, respectively, to define the field dependence of the effective blocking temperature of the NPs' ensemble:

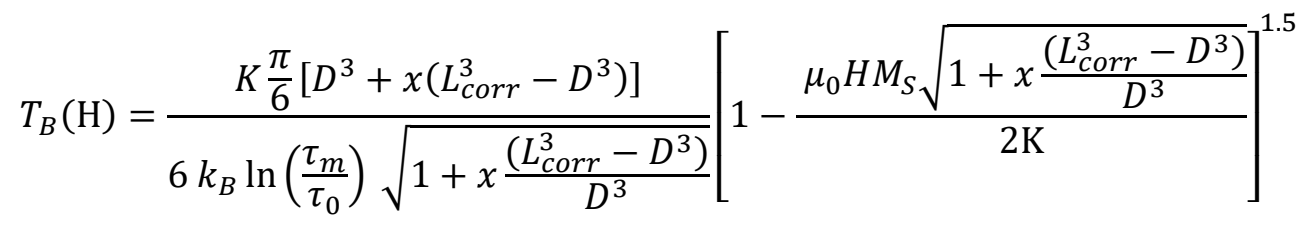

The experimental values of $T_{B}(H)$ were extracted using FC and ZFC curves measured at different applied fields (Figure S4). The $\mathrm{MFC}_{\mathrm{FC}} \mathrm{M}_{\mathrm{ZFC}}$ curve (Figure S5a) allows estimating the effective distribution of energy barriers (Figure S5b) ${ }^{11,12}$ :

$$
f(T) \propto-\frac{d\left(M_{F C}-M_{Z F C}\right)}{d T}
$$

The average $T_{B}$ is conventionally defined as the temperature at which $50 \%$ of the sample is in the superparamagnetic regime ${ }^{11,13}$. This corresponds to the temperature at which the integral of the $f(T)$ reaches $50 \%$ of its maximum value, i.e., the temperature at which the $\left(\mathrm{M}_{\mathrm{FC}}-\mathrm{M}_{\mathrm{ZFC}}\right)$ curve is reduced to half of its value at the lowest temperature.

In the case of significant particle size distribution, de Almeida et al. have recently proposed an alternative expression ${ }^{14}$ :

$$
f(T) \propto-\frac{1}{T} \frac{d\left(M_{F C}-M_{Z F C}\right)}{d T}
$$

This formula uses an additional 1/T term, which aims to renormalize the contribution of the particles with the largest volume, which reverse at higher $T_{B}$ and, due to their larger magnetic moment, they could bias the distribution of blocking temperatures to larger values. From the $-(1 / T)\left[d\left(M_{F C}-M_{Z F C}\right) / d T\right]$ curves (Figure $\left.S 5 c\right)$, the corresponding cumulative distribution function (CDFs) (Figure S5d) are calculated and used to estimate blocking temperature, as the temperature where the CDF reaches $50 \%$ of its maximum value. 
a

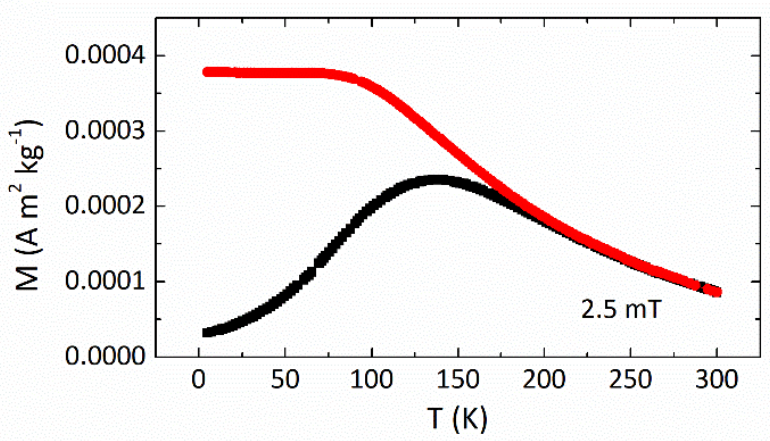

C

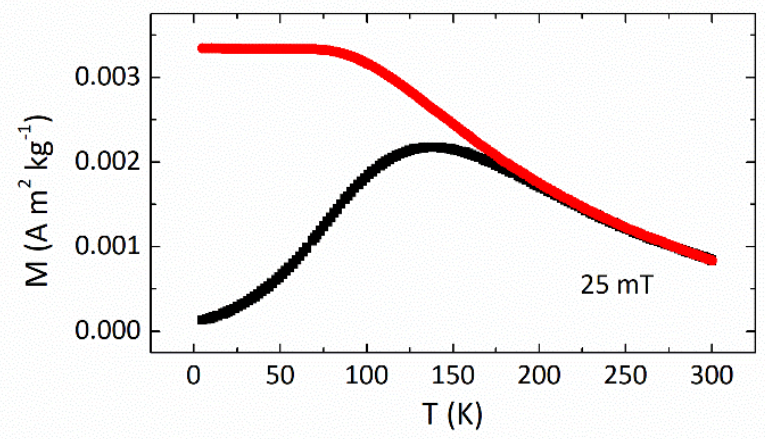

e

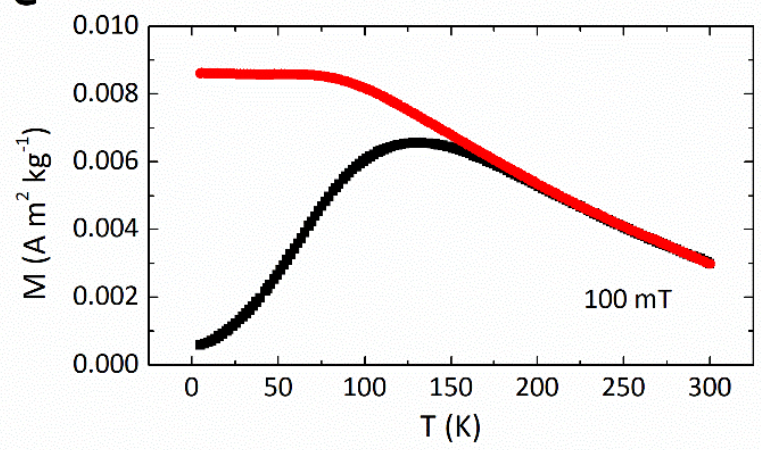

g

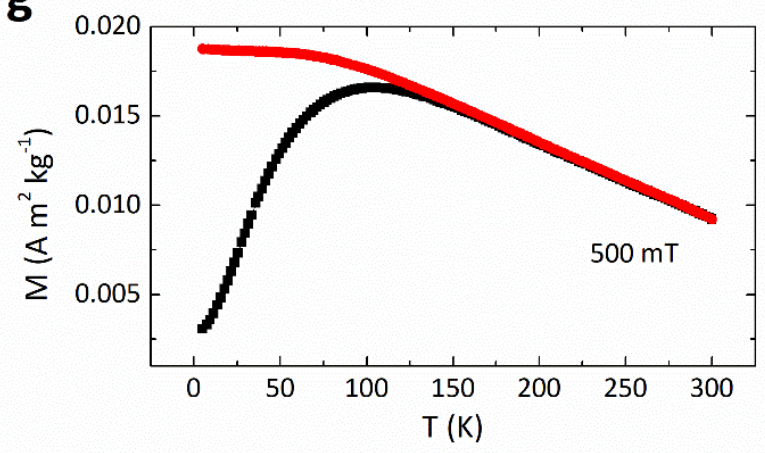

b

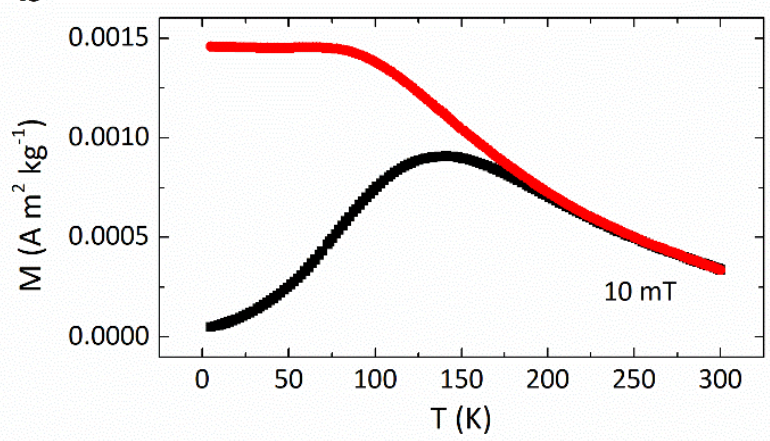

d

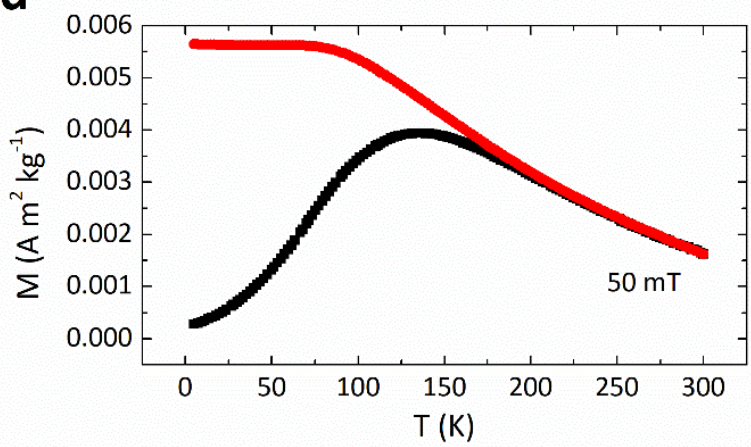

f

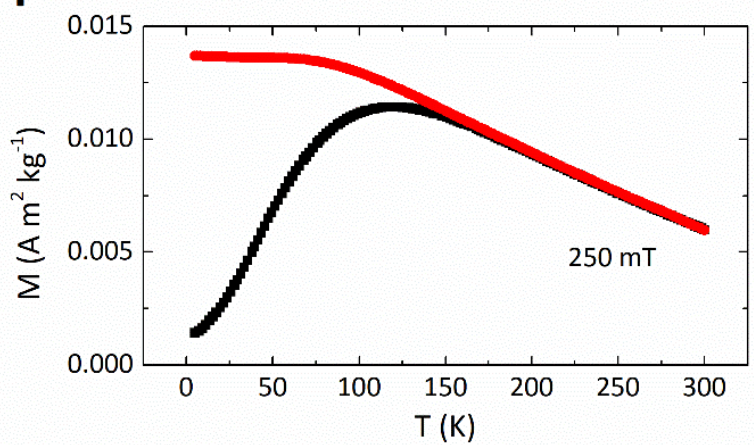

h

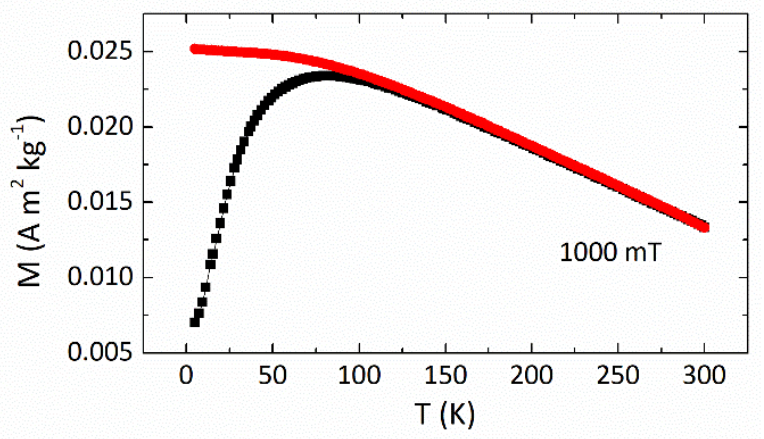

Figure S4. ZFC (black squares) and FC (red circles) measured at different field: (a) $2.5 \mathrm{mT}$, (b) $10 \mathrm{mT}$, (c) $25 \mathrm{mT}$, (d) $50 \mathrm{mT}$, (e) $100 \mathrm{mT}$, (f) $250 \mathrm{mT},(\mathrm{g}) 500 \mathrm{mT}$, and (h) $1000 \mathrm{mT}$. 
a

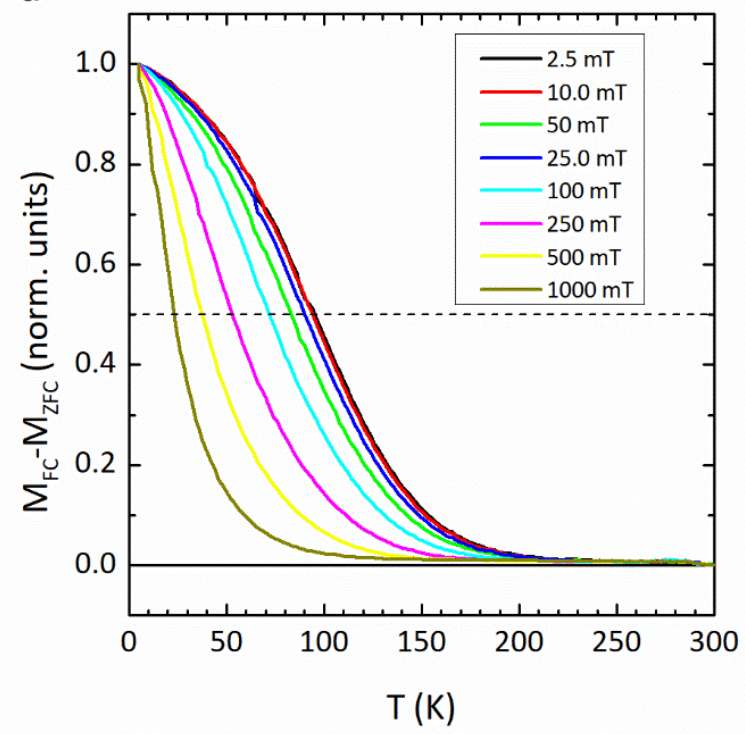

C

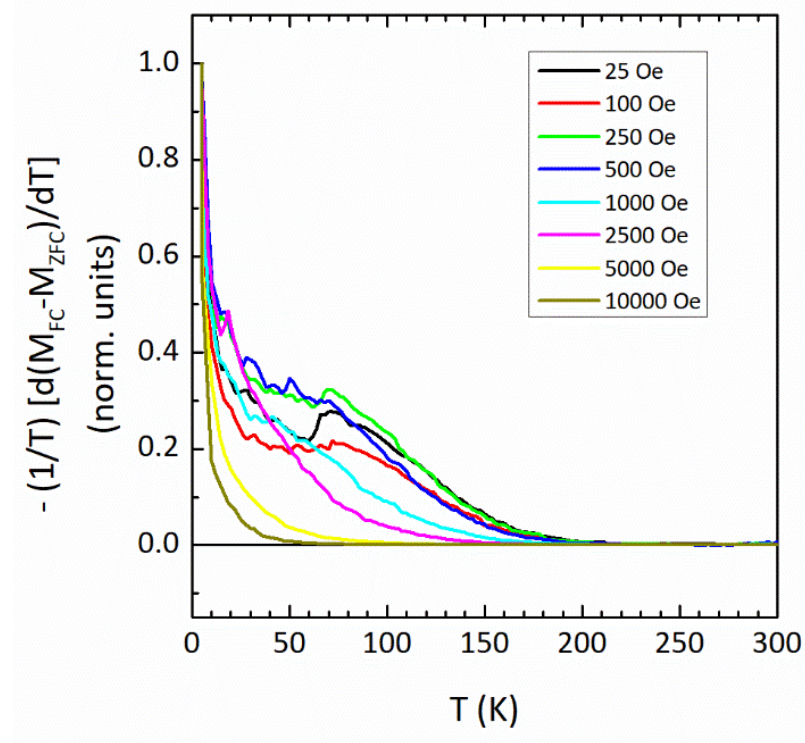

b

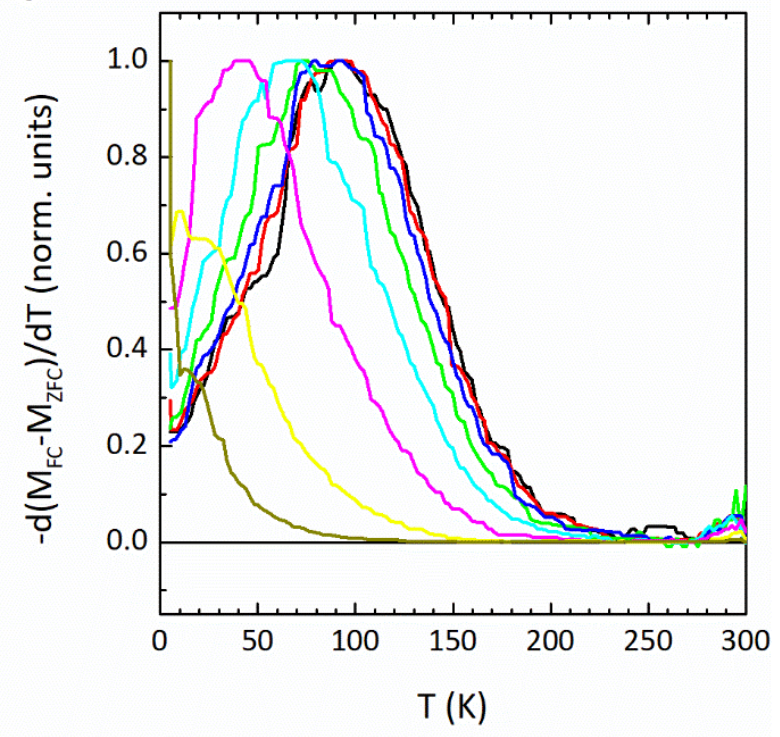

d

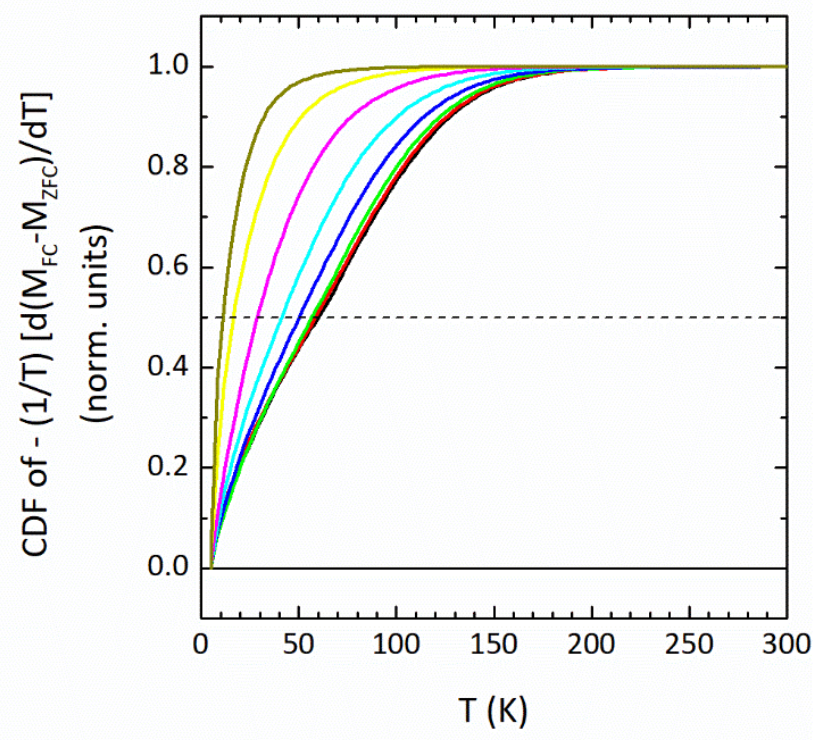

Figure S5. (a) $M_{F C}-M_{Z F C}$ curves as a function of the applied field, (b) the corresponding negative derivative, (c) the corresponding - (1/T) $\left[d\left(M_{F C}-M_{Z F C}\right) / d T\right]$ curves, and (d) their cumulative distribution function (CDF). 


\section{$\Delta$ M-plots}

Isothermal remanent magnetization (IRM) and direct current magnetization (DCD) protocols are two effective tools to investigate interparticle interactions. For non-interacting single-domain particles with uniaxial anisotropy and magnetization reversal by coherent rotation, the IRM and DCD curves are related via the Wohlfarth equation ${ }^{15}$, which was proposed by Kelly et al. ${ }^{16}$ in the form:

$$
\Delta M=m_{D C D}(H)-1+2 m_{I R M}(H)
$$

where $m_{D C D}(H)$ and $m_{I R M}(H)$ represent the reduced terms $M_{D C D}(H) / M_{D C D}\left(H_{\max }\right)$ and $M_{I R M}(H) / M_{I R M}\left(H_{\max }\right)$, with $M_{D C D}\left(H_{\max }\right)$ and $M_{I R M}\left(H_{\max }\right)$ being the remanence values for the DCD and IRM curves for a large reversal field $H_{\max }$ capable of fully saturate the sample. The plot is equal to zero and deviates from this value in case of interactions: positive or negative deviations are indicative of the presence of interactions promoting the magnetized or demagnetized state, respectively.

Unfortunately, the maximum experimental field available $(5 \mathrm{~T})$ was not capable of fully saturating the sample, hence the results of the plot in Figure $\mathbf{S 6}$ do not have absolute quantitative value. Nevertheless, they evidence the presence of a strongly interacting regime with dominant demagnetization effects.

a

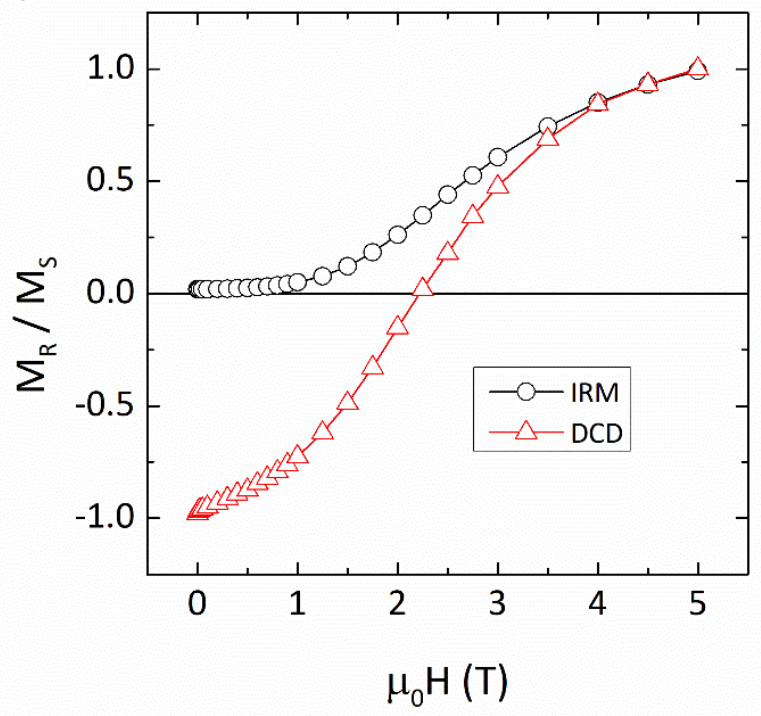

b

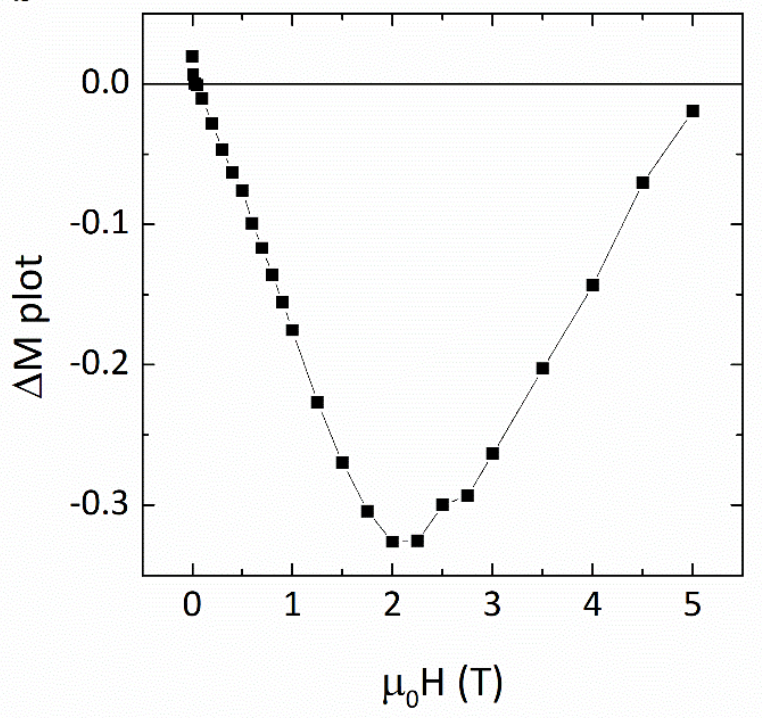

Figure S6. (a) IRM and DCD curves measured at $5 K$ and (b) the corresponding $\triangle M$ plot. 


\section{Low-temperature Magnetization vs Field curves}

Figure S7 shows $\mathrm{M}$ vs $(\mathrm{H})$ curves for the nanocomposite ( $\mathrm{CoFe}_{2} \mathrm{O}_{4}+$ amorphous) and $\mathrm{Co}_{0.25} \mathrm{Fe}_{2.75} \mathrm{O}_{4} \mathrm{NPs}$ synthesized at a $\mathrm{pH}$ value of 9.5 . The $\mathrm{Co}_{0.25} \mathrm{Fe}_{2.75} \mathrm{O}_{4}$ sample presents the highest saturation magnetization $\approx 115$ vs $40 \mathrm{~A} \mathrm{~m}^{2} \mathrm{Kg}^{-1}$. Such high value for the $\mathrm{Co}_{0.25} \mathrm{Fe}_{2.75} \mathrm{O}_{4} \mathrm{NPs}$ can be related to the specific cationic distribution due to the partial substitution of $25 \%$ of $\mathrm{Fe}^{2+}$ with $\mathrm{Co}^{2+}$ ions in the ferrimagnetic spinel structure ${ }^{17}$.

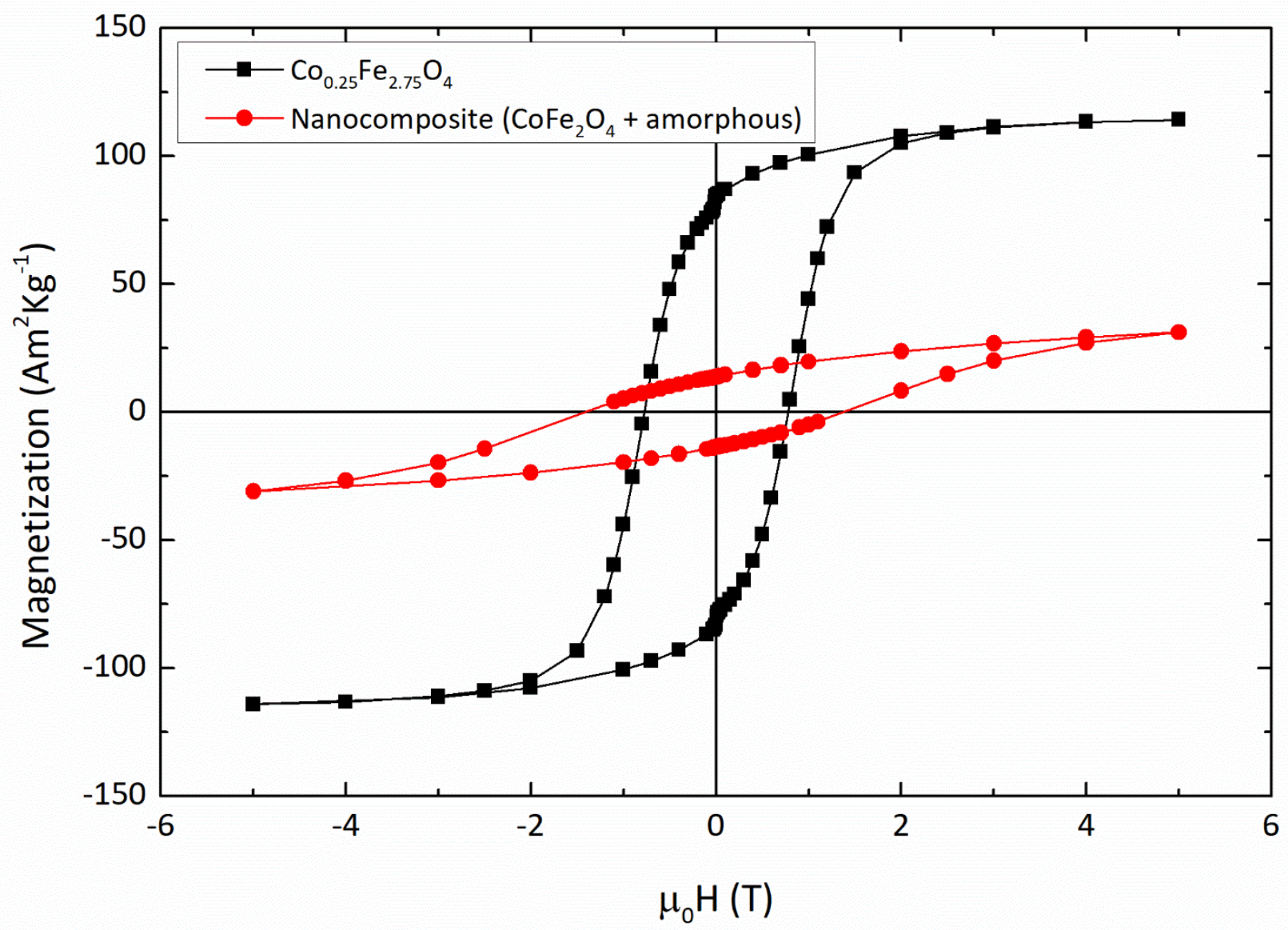

Figure S7: Magnetization vs field curves measured at $5 \mathrm{~K}$ for the nanocomposite $\left(\mathrm{CoFe}_{2} \mathrm{O}_{4}+\right.$ amorphous) and the $\mathrm{Co}_{0.25} \mathrm{Fe}_{2.75} \mathrm{O}_{4} \mathrm{sample}_{\text {. }}$ 


\section{Micromagnetic simulations}

The micromagnetic model of the nanocomposite $\left(\mathrm{CoFe}_{2} \mathrm{O}_{4}+\right.$ amorphous) was implemented using the software Mumax ${ }^{18}$ and validated to reproduce the magnetization vs field curve measured at $5 \mathrm{~K}$. To represent the large aggregates, we have created e a simulation box of $96 \times 96 \times 96 \mathrm{~nm}^{3}$ discretized in cubic cells of $3 \mathrm{~nm}$ edge. Besides, the sample was divided into grains of $10 \mathrm{~nm}$ by Voronoi tessellation. Each grain owns an effective uniaxial anisotropy with a random orientation of easy axis, in a simple random anisotropy model ${ }^{19}$. Within this granular structure, 8192 cells have been used to individually reproduce the embedded $3 \mathrm{~nm}$ crystalline particles, corresponding to $25 \%$ of the total simulated volume, as estimated from the Rietveld refinement of the XRD pattern. The simulated structure is repeated using periodic boundary conditions with 10 copies at each side of the original simulation box, for a total size for the virtual sample of about $2 \times 2 \times 2 \mu \mathrm{m}^{3}$. To improve the statistical representation of the sample, the simulation was repeated 10 times with different random configurations, hence averaging the results. The crystalline phase is described with bulk parameters, i.e., exchange stiffness of $12 \mathrm{pJ} / \mathrm{m}$, saturation magnetization of $85 \mathrm{~A} \mathrm{~m}^{2} \mathrm{~kg}^{-1}$, and cubic anisotropy of $290 \mathrm{KJ} / \mathrm{m}^{3}$, with a random direction of easy axis in each $3 \mathrm{~nm}$ element. For the amorphous phase, the parameters are defined according to the experimental observations collected from structural (XRD, TEM) and magnetic (SQUID magnetometry, Mössbauer spectroscopy) measurements. While the saturation magnetization of the amorphous matrix was not directly experimentally accessible, a starting value has been estimated through the fit of the high field part of the $M(H)$ curve with the law of approach to saturation (LAS). Since the $M(H)$ protocol does not fully saturate the sample, the LAS approach cannot give values of absolute significance. Nevertheless, we have used it as a suggestion for a reasonable interval of confidence to guide the optimization of the value of saturation magnetization of the amorphous fraction. Considering also the canting angle at $8 \mathrm{~T}$ and the relative experimental error, this range was estimated between about 40 and $60 \mathrm{~A} \mathrm{~m}^{2} \mathrm{~kg}^{-1}$. If one considers a fully collinear structure for the crystalline cobalt ferrite fraction and a canted one only for the amorphous matrix, the saturation magnetization of the amorphous matrix $\left(\mathrm{Ms}_{\mathrm{s}}^{\text {matrix }}\right)$ should be between $\approx 30$ and $50 \mathrm{~A} \mathrm{~m}^{2} \mathrm{~kg}^{-1}$. Hence, the only real free parameter was the magnetic anisotropy, optimized with $\mathrm{Ms}_{s}{ }^{\text {matrix }}$ to reproduce the experimental $\mathrm{M}(\mathrm{H})$ curve recorded at $5 \mathrm{~K}$. The best result was obtained setting $\mathrm{Ms}^{\text {matrix }}=38 \mathrm{~A} \mathrm{~m}^{2} \mathrm{~kg}^{-1}$, and using a log-normal distribution of magnetic anisotropy values with a mean of $3.0 \mathrm{MJ} / \mathrm{m}^{3}$ and standard deviation of $2.0 \mathrm{MJ} / \mathrm{m}^{3}$, resulting in a mode of $1.7 \mathrm{MJ} / \mathrm{m}^{3}$ (Figure S8).

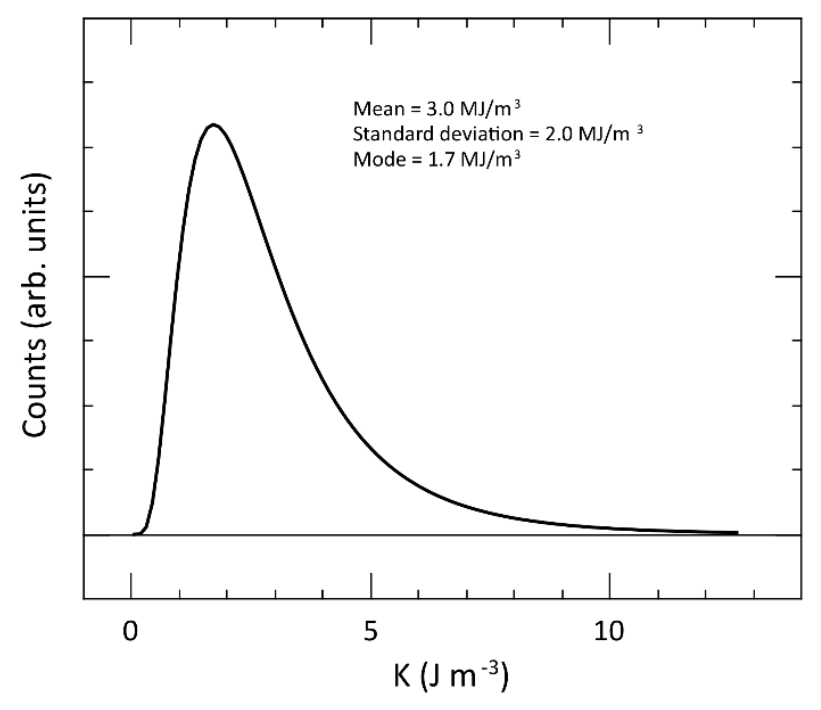

Figure S8. Optimized anisotropy constant distribution for the amorphous grainy matrix. 
As further validation of the model, we have simulated the system at $8 \mathrm{~T}$ (Figure S9). It shows that the sample still does not reach saturation but, most importantly it allows calculating an average canting angle of $40(2)^{\circ}$, in good agreement with the experimental Mössbauer data and further reinforcing the parameters used in the model for the amorphous component.

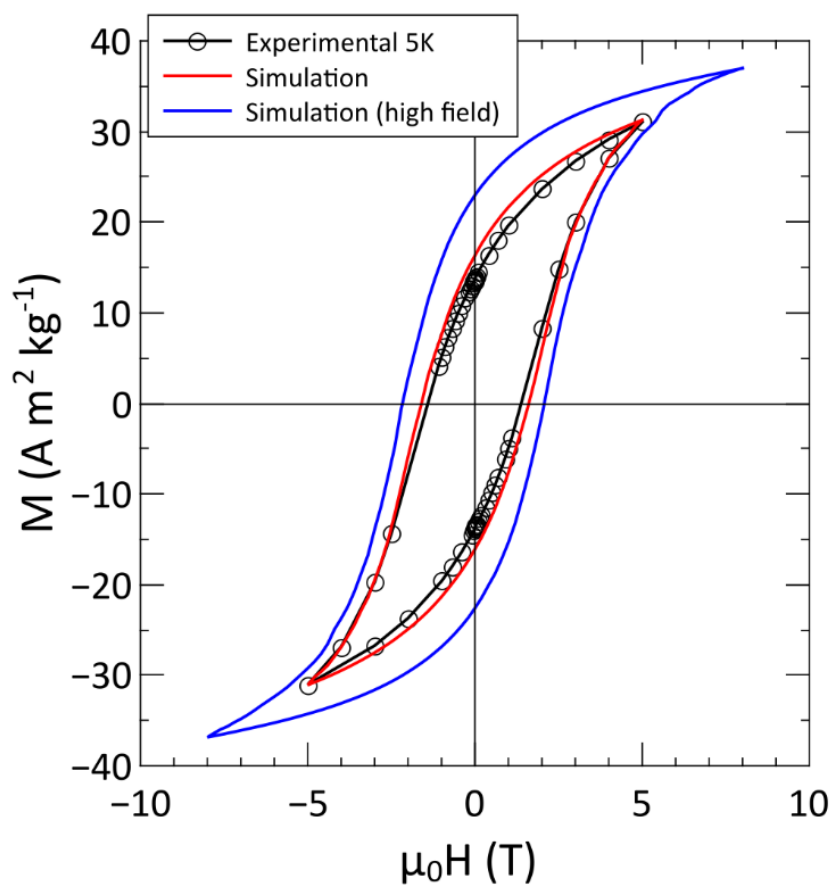

Figure S9. Comparison of the experimental curve measured in the interval +/- $5 T$ (black lines and circles), the corresponding simulated curve (red line), and a simulation carried out in an interval of $+/-8 \mathrm{~T}$ (blue line).

To investigate the role of the coupling among each component, we have performed simulations individually removing each exchange contribution and dipolar interactions (Figure S10). Removing the exchange coupling among the matrix grains or removing the exchange among the crystalline elements, as well as the dipolar fields, has minimal effects. In all the cases, the coercivity has a slight tendency to increase, since all three interactions have a weak effect on reducing the role of the high anisotropy of the individual amorphous grains, averaging down the effective anisotropy value (Figure S10a). On the other hand, the exchange between crystalline and amorphous elements plays the main role. With its strong magnitude, it induces a single-phase magnetic behavior. In the absence of this exchange, a double phase magnetization reversal is strongly evident, with a low reversal field for the crystalline grains and only partial reversal of the matrix in the range of magnetic field used (Figure S10a).

To confirm the validity of the model, we have also included a simulation in which the large anisotropy is attributed to the crystalline component and the low bulk-like value used for the matrix. As visible in Figure S10b, this will lead to an easy saturation at $5 \mathrm{~T}$ with no residual canted structure. On the other hand, the low fraction of crystalline elements will not lead to the large coercivity measured, despite the large value of anisotropy used, compatible with what experimentally observed for similar individual particles with large surface anisotropy ${ }^{20}$. 

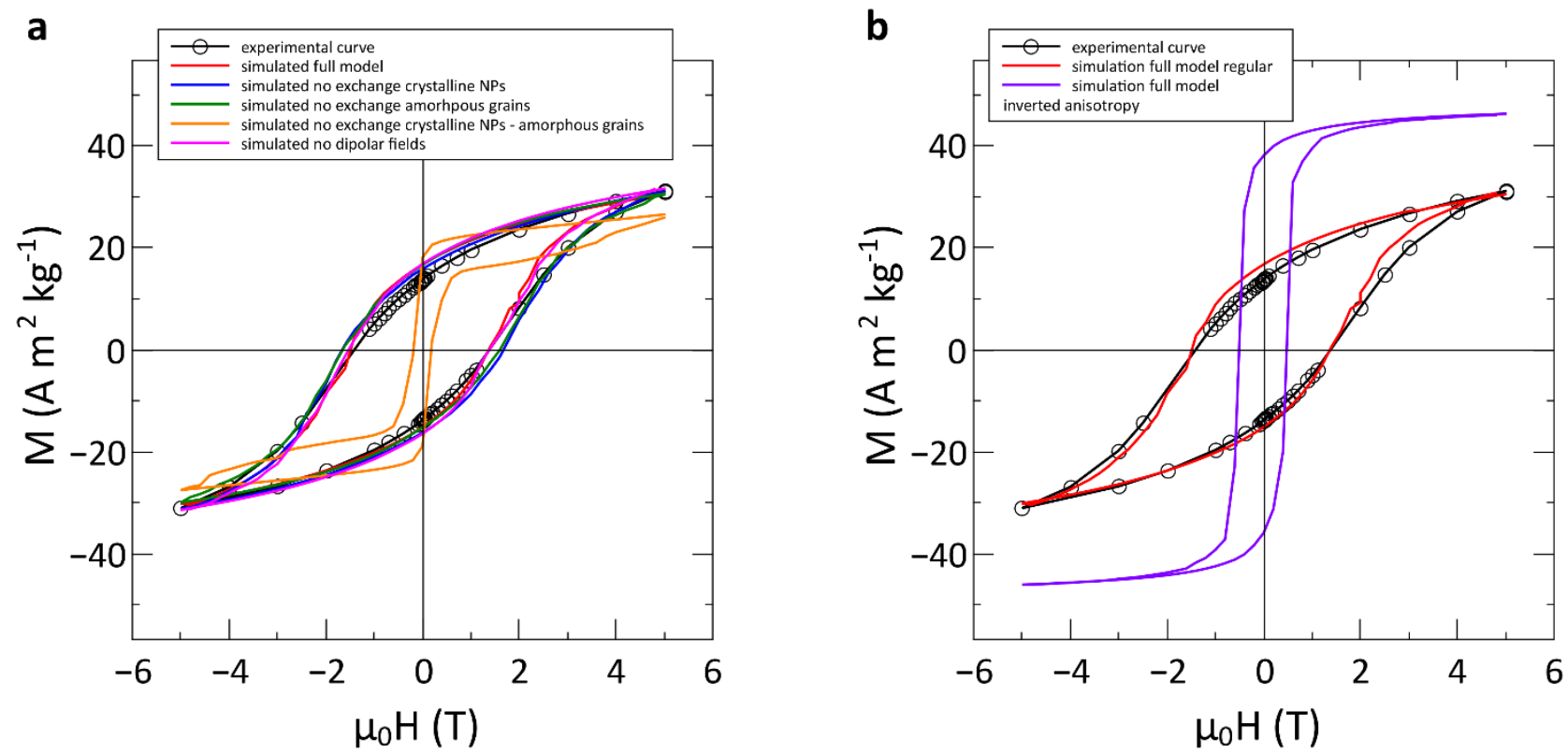

Figure S10. (a) The experimental curve (black circles and line) is compared to the simulated one using the full model (red line) and with removing exchange contribution among crystalline elements (blue line), among amorphous grains (green line), between crystalline and amorphous components (orange line), and turning off the dipolar fields (magenta line). (b) The experimental curve (black circles and line) is compared with the simulated one using the full model (red line) and an equivalent one with inverted anisotropy between the crystalline and amorphous phase (purple line). 


\section{References}

(1) Fernandes de Medeiros, I. A.; Lopes-Moriyama, A. L.; de Souza, C. P. Effect of Synthesis Parameters on the Size of Cobalt Ferrite Crystallite. Ceram. Int. 2017, 43 (5), 3962-3969. https://doi.org/10.1016/j.ceramint.2016.10.105.

(2) Zhao, D.; Wu, X.; Guan, H.; Han, E. Study on Supercritical Hydrothermal Synthesis of CoFe2O4 Nanoparticles. J. Supercrit. Fluids 2007, 42 (2), 226-233. https://doi.org/10.1016/j.supflu.2007.03.004.

(3) Tronc, E.; Prené, P.; Jolivet, J. P.; Dormann, J. L.; Grenèche, J. M. Spin Canting in Y-Fe2O3 Nanoparticles. Hyperfine Interact. 1998, 112 (1), 97-100. https://doi.org/10.1023/A:1011092712136.

(4) Coey, J. M. D. Noncollinear Spin Arrangement in Ultrafine Ferrimagnetic Crystallites. Phys. Rev. Lett. 1971, 27 (17), 1140-1142. https://doi.org/10.1103/PhysRevLett.27.1140.

(5) Herzer, G. The Random Anisotropy Model. In Properties and Applications of Nanocrystalline Alloys from Amorphous Precursors; Idzikowski, B., Švec, P., Miglierini, M., Eds.; NATO Science Series; Springer-Verlag: Berlin/Heidelberg, 2005; Vol. 184, pp 15-34. https://doi.org/10.1007/1-4020-2965-9_2.

(6) Muscas, G.; Concas, G.; Laureti, S.; Testa, A. M.; Mathieu, R.; De Toro, J. A.; Cannas, C.; Musinu, A.; Novak, M. A.; Sangregorio, C.; Lee, S. S.; Peddis, D. The Interplay between Single Particle Anisotropy and Interparticle Interactions in Ensembles of Magnetic Nanoparticles. Phys. Chem. Chem. Phys. 2018, 20 (45), 28634-28643. https://doi.org/10.1039/C8CP03934H.

(7) Knobel, M.; Nunes, W. C.; Winnischofer, H.; Rocha, T. C. R.; Socolovsky, L. M.; Mayorga, C. L.; Zanchet, D. Effects of Magnetic Interparticle Coupling on the Blocking Temperature of Ferromagnetic Nanoparticle Arrays. J. Non. Cryst. Solids 2007, 353 (8-10), 743-747. https://doi.org/10.1016/j.jnoncrysol.2006.12.037.

(8) Nunes, W. C.; Socolovsky, L. M.; Denardin, J. C.; Cebollada, F.; Brandl, A. L.; Knobel, M. Role of Magnetic Interparticle Coupling on the Field Dependence of the Superparamagnetic Relaxation Time. Phys. Rev. B 2005, 72 (21), 212413. https://doi.org/10.1103/PhysRevB.72.212413.

(9) Weissmüller, J.; Michels, A.; Barker, J. G.; Wiedenmann, A.; Erb, U.; Shull, R. D. Analysis of the Small-Angle Neutron Scattering of Nanocrystalline Ferromagnets Using a Micromagnetics Model. Phys. Rev. B 2001, 63 (21), 214414. https://doi.org/10.1103/PhysRevB.63.214414.

(10) Michels, a.; Viswanath, R.; Barker, J.; Birringer, R.; Weissmüller, J. Range of Magnetic Correlations in Nanocrystalline Soft Magnets. Phys. Rev. Lett. 2003, 91 (26), 267204. https://doi.org/10.1103/PhysRevLett.91.267204.

(11) Bruvera, I. J.; Mendoza Zélis, P.; Pilar Calatayud, M.; Goya, G. F.; Sánchez, F. H. Determination of the Blocking Temperature of Magnetic Nanoparticles: The Good, the Bad, and the Ugly. J. Appl. Phys. 2015, 118 (18), 184304. https://doi.org/10.1063/1.4935484.

(12) Concas, G.; Congiu, F.; Muscas, G.; Peddis, D. Determination of Blocking Temperature in Magnetization and Mössbauer Time Scale: A Functional Form Approach. J. Phys. Chem. C 2017, 121 (30), 16541-16548. https://doi.org/10.1021/acs.jpcc.7b01748.

(13) Mamiya, H.; Ohnuma, M.; Nakatani, I.; Furubayashim, T. Extraction of Blocking Temperature Distribution from Zero-Field-Cooled and Field-Cooled Magnetization Curves. IEEE Trans. Magn. 2005, 41 (10), 3394- 
3396. https://doi.org/10.1109/TMAG.2005.855205.

(14) de Almeida, A. A.; De Biasi, E.; Mansilla, M. V.; Valdés, D. P.; Troiani, H. E.; Urretavizcaya, G.; Torres, T. E.; Rodríguez, L. M.; Fregenal, D. E.; Bernardi, G. C.; Winkler, E. L.; Goya, G. F.; Zysler, R. D.; Lima, E. Magnetic Hyperthermia Experiments with Magnetic Nanoparticles in Clarified Butter Oil and Paraffin: A Thermodynamic Analysis. J. Phys. Chem. C 2020, 124 (50), 27709-27721. https://doi.org/10.1021/acs.jpcc.0c06843.

(15) Wohlfarth, E. P. Relations between Different Modes of Acquisition of the Remanent Magnetization of Ferromagnetic Particles. J. Appl. Phys. 1958, 29 (3), 595-596. https://doi.org/10.1063/1.1723232.

(16) Kelly, P. E.; O’Grady, K.; Mayo, P. L.; Chantrell, R. W. Switching Mechanisms in Cobalt-Phosphorus Thin Films. IEEE Trans. Magn. 1989, 25 (5), 3881-3883. https://doi.org/10.1109/20.42466.

(17) Ayyappan, S.; Mahadevan, S.; Chandramohan, P.; Srinivasan, M. P.; Philip, J.; Raj, B. Influence of Co 2+ Ion Concentration on the Size, Magnetic Properties, and Purity of CoFe 204 Spinel Ferrite Nanoparticles. J. Phys. Chem. C 2010, 114 (14), 6334-6341. https://doi.org/10.1021/jp911966p.

(18) Vansteenkiste, A.; Leliaert, J.; Dvornik, M.; Helsen, M.; Garcia-Sanchez, F.; Van Waeyenberge, B. The Design and Verification of MuMax3. AIP Adv. 2014, 4 (10), 107133. https://doi.org/10.1063/1.4899186.

(19) Herzer, G. The Random Anisotropy Model - A Critical Review and Update. In Properties and Applications of Nanocrystalline Alloys from Amorphous Precursors; Idzikowski, B., Švec, P., Miglierini, M., Eds.; NATO Science Series; Springer Netherlands: Berlin/Heidelberg, 2005; Vol. 184, pp 15-34. https://doi.org/10.1007/1-4020-2965-9_2.

(20) Peddis, D.; Mansilla, M. V; Mørup, S.; Cannas, C.; Musinu, A.; Piccaluga, G.; D’Orazio, F.; Lucari, F.; Fiorani, D.; D'Orazio, F. Spin-Canting and Magnetic Anisotropy in Ultrasmall CoFe2O4 Nanoparticles. J. Phys. Chem. B 2008, 112 (29), 8507-8513. https://doi.org/10.1021/jp8016634. 